\title{
An affordable methodology for quantifying waterborne microplastics - an emerging contaminant in inland-waters
}

\author{
Christian Dunn, ${ }^{*}$ Jedd Owens, ${ }^{1}$ Luke Fears, ${ }^{1}$ Laura Nunnerley, ${ }^{1}$ Julian Kirby, ${ }^{2}$ Oliver L. Armstrong, ${ }^{1}$ P. John Thomas, ${ }^{1}$ \\ Dan Aberg, ${ }^{1}$ William Gilder, ${ }^{1}$ Dannielle Green, ${ }^{3}$ Rachael Antwis, ${ }^{4}$ Chris Freeman ${ }^{1}$ \\ ${ }^{1}$ School of Natural Sciences, Bangor University, Deiniol Road, Bangor, Gwynedd LL57 2UW; ${ }^{2}$ Friends of the Earth, The Printworks, \\ 139 Clapham Road, London SW9 0HP; ${ }^{3}$ School of Life Sciences, Faculty of Science and Engineering, Anglia Ruskin University, \\ Cambridge Campus, Cambridge CB1 1PT; ${ }^{4}$ School of Environment and Life Sciences, Salford University, Salford M5 4WT, UK
}

\begin{abstract}
The occurrence of microplastics in marine habitats is well documented and of growing concern. The presence of these small $(<5$ $\mathrm{mm}$ ) pieces of plastic is less well recorded in inland water systems. In this paper, we determine a cost-efficient and straightforward method for the collection and identification of microplastics in UK inland waters. We found pieces of microplastic from all sample sites ranging from over $1000 \mathrm{~L}^{-1}$ in the River Tame, to $2.4 \mathrm{~L}^{-1}$ in Loch Lomond. The presence of microplastics in all waters tested suggests it should now be classed as an emergent contaminant, with routine monitoring required.
\end{abstract}

\section{INTRODUCTION}

Microplastics are pieces of plastic less than $5 \mathrm{~mm}$ in size (Thompson et al., 2009) that come from a variety of either primary or secondary sources. Primary sources are those plastics purposefully manufactured to such a size for use in cosmetics or cleaning products, or as part of the general plastic production system. Secondary sources of microplastics are those fragments of plastic produced through the breakdown of larger pieces. Both types can enter inland water systems through a variety of ways and the full impacts of these pellets, fragments and fibres on ecosystems, wildlife and indeed our own health are not yet fully understood (Cole et al., 2011; Eerkes-Medrano et al., 2015; de Souza Machado, 2017).

Some pollutants in inland waters are regularly monitored and guidelines are enforced to ensure levels do not exceed beyond stated "safe" concentrations. Other pollutants - so called emergent contaminants - such as

Corresponding author: c.dunn@bangor.ac.uk

Key words: Microplastics; plastic pollution; fluorescence microscopy; emergent contaminants; inland waters.

Edited by: Gianluca Corno, CNR-IRSA Verbania, Italy.

Received: 28 June 2019

Accepted: 7 November 2019.

This work is licensed under a Creative Commons Attribution NonCommercial 4.0 License (CC BY-NC 4.0).

${ }^{\circ}$ Copyright: the Author(s), 2019

Licensee PAGEPress, Italy

J. Limnol., 2020; 79(1): 92-96

DOI: 10.4081/jlimnol.2019.1943 pharmaceutical waste, personal care products and illicit drugs are only just being recognised as issues, and work is being conducted to investigate these problems fully (Tran et al., 2018). In this paper, we use a practical and inexpensive method to highlight the widespread presence of microplastics in UK mainland waters, indicating it is essential they are now considered an important emergent contaminant. The methodology described purposefully only uses standard laboratory equipment and a commercially available fluorescence light attachment, offering the potential of this method being used to acquire data on many more sites on a regular basis, by a wide variety of organisations and collaborators.

\section{METHODS}

Four, clean, one-litre glass amber bottles with plastic lids, with a standard $(\sim 2.5 \mathrm{~cm})$ opening, were rinsed thoroughly with water from the sample site (see Tab. 1 for a full list of the sites). Each bottle was then filled to the very top with site water from a depth of approximately 5 $-10 \mathrm{~cm}$ from the surface, and capped underwater (Green et al., 2018). The sampler remained downstream of the bottle being filled at all times, and water was collected from a safe wading distance and sampling was conducted between June 2018 and February 2019. Samples were stored in the laboratory at $4^{\circ} \mathrm{C}$ in the dark, until analysis was completed.

The contents of each bottle were filtered using a glass vacuum-pump filtering system through a $\mathrm{GF} / \mathrm{C}$ glass filter $\left(1.2 \mu \mathrm{m}\right.$ pore size; GE Healthcare Whatman $\left.{ }^{\mathrm{TM}}\right)$ - chosen due to their relative affordability. Filters were dried and analysed for microplastic numbers and types (HidalgoRuz et al., 2012; Ravit et al., 2017) using a dissecting microscope (with a magnification level ranging from 10 to 40 ) using either a standard visible light system or a 
fluorescence lighting system attached to the same microscope. For this research a NIGHTSEA ${ }^{\mathrm{TM}}$ Stereo Microscope Fluorescence Adapter was used. The designation was Royal Blue (RB) with an excitation of 440-460 $\mathrm{nm}$ and emission filter of $500 \mathrm{~nm}$ (longpass). This set-up was chosen as the adaptor fits to any standard dissection microscope and is low-cost ( 1000 USD) compared to specialised fluorescence microscope set-ups used in previous studies (Qui et al., 2015). Comparisons were conducted to determine which lighting system (visible or fluorescence) made it easier to detect microplastics. When particles could not be visually identified as microplastic suspected pieces were tested using the bending test (to see if they snapped or, if they were plastic, bent) and hot-needle technique (Hurley et $a l ., 2018)$. The latter involves placing a very hot needle or pin near a suspected piece of plastic. If it is plastic, it will melt or curl.

Measures were taken to minimise the contamination of water and filters throughout the procedure. Filters were kept in glass petri-dishes and only uncovered for the necessary analysis, such as the hot-pin method. Lab coats were regularly cleaned with lint removers. A series of control samples were made by using the same type and manufacturer of bottles and lids, following the usual filtering process but using pre-filtered water - where necessary, results from these controls were subtracted from the experimental samples to give a set of normalised results. These controls were conducted during any filtering and sampling period in the laboratory. Additional precautions to ensure robustness of the controls were also adhered to, such as leaving the vacuum pump system on for approximately the same time as it took the inland water samples to go through the filter.

Each sample was counted four times by a trained observer, and the samples were counted in a randomised order to help prevent bias. An average of each count was taken as the result for that sample. For samples that contained many pieces of microplastic, when observers had counted 1000 single pieces of microplastic (all types combined) they stopped counting and the result was recorded as "> $>1000$ ".

Due to the equipment used and identification process implemented all microplastic pieces counted were longer than approximately $200 \mu \mathrm{m}$ and wider than around $50 \mu \mathrm{m}$, or had an area greater than approximately $2500 \mu \mathrm{m}$.

Significant differences between results were determined by an independent samples $t$-test, using IBM SPSS Statistics 25, after checking the usual assumptions of parametric tests were not violated.

Using the fluorescence lighting system attached to the dissecting microscope produced greater detection levels of microplastics than just using a standard, visible light system; especially for identifying fragments. For instance, there was a statistically significant difference when looking at fragments under the two different lighting conditions for Afon Cegin (the site used for most of the method development): $t(6.27)=5.07, \mathrm{P}=0.001$. The average count for microplastics in one litre of water observed under the standard lighting system was 22.3 (standard error, $\mathrm{SE}=3.4$ ) compared to a mean average of $73.7(\mathrm{SE}=9.5)$ using fluorescence lighting.

The advantage of using fluorescence can be seen in Fig. 1: the microplastic fragment cannot be observed on the filter paper in Fig. 1A, taken under normal lighting conditions, whilst it is clearly visible on the same filter paper in Fig. 1B for which the fluorescence system is used (the same for Fig. 1 C,D). However, as some organics and minerals present in the samples also fluoresce, it is vital that switching between the two lighting sources is considered and standard techniques, e.g. bending test, hotpin method, Raman spectroscopy (Araujo et al., 2018) are used to be sure of microplastic identification.

\section{RESULTS}

All sites analysed had microplastics present (Tab. 1). Loch Lomond had the lowest number of microplastics with a total of 2.4 pieces per litre $\left(\mathrm{L}^{-1}\right)$ while River Tame had the most with over 1000 pieces $\mathrm{L}^{-1}$. As with all reported concentrations of microplastics this is the normalised result, following the subtraction of a set of pre-filtered control, or 'blank', samples. However, to
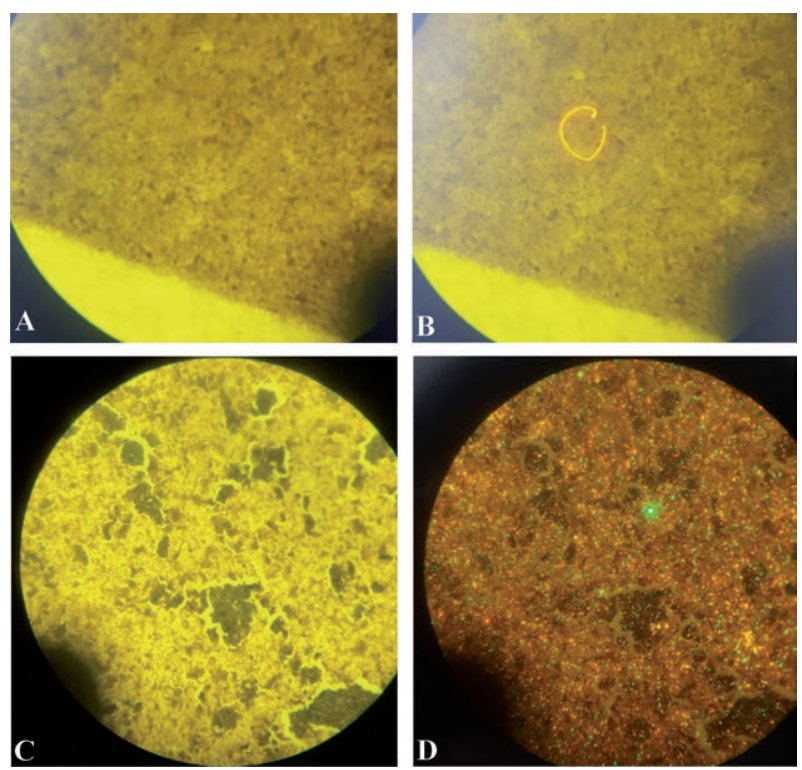

Fig. 1. A section of filter paper looked at through $x 40$ magnification for microplastic identification. a,c) Photographed using only standard, visible light; b,d) photographed using a fluorescence lighting system. 
Tab. 1. Numbers and types of microplastic found in inland waters from the UK. Results are a mean average ( $\mathrm{n}=4$ for all sites except Ullswater and Afon Cegin, $n=6$ ) from one litre of site water. Microplastics were categorised as fibre (pieces of line or filament), fragment (pieces broken off from larger plastics), film (breakdown from bags, wrappers etc), pellet (microbeads and nurdles), or foam (broken pieces of polystyrene items). \pm indicates standard error (there is no standard error for River Tame due to the count being recorded as $>1000$ - other microplastic types may also have been present but the methodology dictates counting stops after 1000 individual microplastic pieces are detected). Numbers in parentheses indicate mean averages $(n=4)$ of procedural blanks, consisting of pre-filtered water. These averages were subtracted from the all relevant sample counts before averaging and statistical analysis.

\begin{tabular}{|c|c|c|c|c|c|c|c|}
\hline \multirow[t]{2}{*}{ Site } & \multirow[t]{2}{*}{ Location } & \multicolumn{5}{|c|}{ Microplastic type } & \multirow[t]{2}{*}{ Total } \\
\hline & & Fibre & Fragment & Film & Pellet & Foam & \\
\hline River Thames & $51^{\circ} 30^{\prime} 30.7^{\prime \prime} \mathrm{N} 0^{\circ} 06^{\prime} 37.0^{\prime \prime} \mathrm{W}$ & $\mathbf{6 . 9} \pm 1.5(0.25)$ & $\mathbf{7 4 . 4} \pm 11(1.75)$ & $\mathbf{1 . 1} \pm 0.4$ & $\mathbf{0 . 1} \pm 0.1$ & $\mathbf{1 . 7} \pm 0.9$ & 84.1 \\
\hline Chester reedbed & $53^{\circ} 12^{\prime} 28.6^{\prime \prime} \mathrm{N} 2^{\circ} 54^{\prime} 12.0^{\prime \prime} \mathrm{W}$ & $\mathbf{1 . 8} \pm 1.9$ & $4.3 \pm 0.3(0.25)$ & $\mathbf{0 . 1} \pm 0.1$ & $\mathbf{0 . 4} \pm 0.4$ & $\mathbf{1 . 1} \pm 0.2$ & 7.6 \\
\hline Ullswater & $54^{\circ} 34^{\prime} 30.4^{\prime \prime} \mathrm{N} 2^{\circ} 54^{\prime} 29.4^{\prime \prime} \mathrm{W}$ & $\mathbf{5} \pm 0.5(0.75)$ & $\mathbf{1 4} \pm 1.4(1)$ & $\mathbf{3 . 3} \pm 0.4$ & $\mathbf{4 . 9} \pm 0.9$ & $\mathbf{2 . 5} \pm 0.4$ & 29.5 \\
\hline River Irwell & $53^{\circ} 29^{\prime} 19.2^{\prime \prime} \mathrm{N} 2^{\circ} 16^{\prime} 07.9^{\prime \prime} \mathrm{W}$ & $\mathbf{0}(1.6)$ & $\mathbf{8 4 . 8} \pm 31.7(13.1)$ & $\mathbf{0}(0.1)$ & $\mathbf{0}(0.1)$ & $\mathbf{0}$ & 84.8 \\
\hline River Tame & $53^{\circ} 27^{\prime} 44.6{ }^{\prime \prime} \mathrm{N} 2^{\circ} 06^{\prime} 03.9^{\prime \prime} \mathrm{W}$ & $\mathbf{0}(1.6)$ & $>\mathbf{1 0 0 0}(13.1)$ & $\mathbf{0}(0.1)$ & $\mathbf{0}(0.1)$ & $\mathbf{0}$ & $>1000$ \\
\hline River Blackwater & $51^{\circ} 43^{\prime} 34.99^{\prime \prime} 0^{\circ} 45^{\prime} 23.7{ }^{\prime \prime} \mathrm{E}$ & $3 \pm 0.2$ & $\mathbf{1 0 . 7} \pm 3(1.5)$ & $\mathbf{0}$ & $\mathbf{0}$ & $\mathbf{1 . 4} \pm 0.8$ & 15.1 \\
\hline Falls of Dochart & $56^{\circ} 27^{\prime} 45.2^{\prime \prime} \mathrm{N} 4^{\circ} 19^{\prime} 13.2^{\prime \prime} \mathrm{W}$ & $\mathbf{1 . 1} \pm 0.5$ & $2.2 \pm 0.7(0.4)$ & $\mathbf{0}$ & $\mathbf{0}$ & $\mathbf{0}$ & 3.3 \\
\hline Loch Lomond & $56^{\circ} 06^{\prime} 43.9^{\prime \prime} \mathrm{N} 4^{\circ} 37^{\prime} 25.8^{\prime \prime} \mathrm{W}$ & $\mathbf{0 . 9} \pm 0.4$ & $\mathbf{1 . 5} \pm 0.6(0.3)$ & $\mathbf{0}$ & $\mathbf{0}$ & $\mathbf{0}$ & 2.4 \\
\hline Afon Cegin & $53^{\circ} 13^{\prime} 53.3^{\prime \prime} \mathrm{N} 4^{\circ} 06^{\prime} 39.4^{\prime \prime} \mathrm{W}$ & $\mathbf{1 4 . 8} \pm 5.7(16)$ & $49.7 \pm 9.5(24)$ & $\mathbf{5 . 7} \pm 3.3(1)$ & $2.7 \pm 1.1(1)$ & $4 \pm 3.4(5)$ & 76.9 \\
\hline Llyn Cefni & $53^{\circ} 16^{\prime} 12.4^{\prime \prime} \mathrm{N} 4^{\circ} 20^{\prime} 22.4^{\prime \prime} \mathrm{W}$ & $7.4 \pm 1.1(0.5)$ & $\mathbf{1 6 . 8} \pm 4.1(1)$ & $7.7 \pm 1.2$ & $8.5 \pm 2$ & $\mathbf{2 . 9} \pm 1.4$ & 43.2 \\
\hline
\end{tabular}

ensure validity of the method it was first checked to confirm the non-normalised results were statistically different to the control samples e.g. for Loch Lomond there was a mean average of $2.4(\mathrm{SE}=0.3)$ pieces of microplastic fragment in the raw samples, and an average of $0.3(\mathrm{SE}=0.5)$ in the controls; showing a statistically significant difference $t(21.45)=3.99, \mathrm{P}<0.001$.

\section{DISCUSSION}

With every sample tested showing evidence of plastics - the most common type across all sites being fragments - it is suggested microplastic pollution is now endemic across all inland water systems in mainland UK. We found it in major rivers running through large urban regions, such as the Thames, Tame and Irwell, as well as remote rivers (Falls of Dochart); wetlands (Chester reedbed); lakes and lochs (Ullswater and Lomond), and reservoirs (Cefni). Some of these are iconic British water systems, and they now all contain microplastic pollution.

There are other methods for measuring microplastic concentrations from the environment and these can have advantages. However, our results illustrate a low-cost, low-tech method for sampling and quantifying microplastic contamination. The process is also relatively time efficient, with it taking approximately 10 to 30 minutes to count the plastic particles visible on most filter papers. There are clear limitations to our affordable and efficient methodology, and not all types of plastic will fluoresce under such conditions (Qiu et al., 2015), so eventually standardised spectroscopic and/or chromatographic methods (imaging FT-IR, microscopy, pyrolysis GC-MS) may become available, and affordable, for higher throughput of microplastic analysis in environmental samples. However, until that time regular monitoring of water systems using the methods outlined in this paper should become routine, as our findings suggest microplastics are now an emergent contaminant.

\section{CONCLUSIONS}

Further work is now essential to investigate fully the health risks of microplastics - to humans and ecosystems - so that "safe" levels can be ascertained, and removal or mitigation processes can be put in place. This could involve the development and use of ecological engineering initiatives such as specially designed constructed treatment wetlands (CTWs) to filter-out plastic particles.

\section{REFERENCES}

Araujo CF, Nolasco, MM, Ribeiro AMP, Ribeiro-Claro PJA, 2018. Identification of microplastics using Raman spectroscopy: Latest developments and future prospects. Water Res. 142:426-440.

Cole M, Lindeque P, Halsband C, Galloway TS, 2011. Microplastics as contaminants in the marine environment: a review. Mar. Pollut. Bull. 62:2588-2597.

Green DS, Kregting L, Boots B, Blockley DJ, Brickle P, da Costa M, Crowley Q, 2018. A comparison of sampling methods for seawater microplastics and a first report of the microplastic litter in coastal waters of Ascension and Falkland Islands. Mar. Pollut. Bull. 137:695-701. 
Hidalgo-Ruz V, Gutow L, Thompson RC, Thiel M, 2012. Microplastics in the marine environment: A review of the methods used for identification and quantification. Environ. Sci. Technol. 46:3060-3075.

Hurley R, Woodward J, Rothwell JJ, 2018. Microplastic contamination of river beds significantly reduced by catchment-wide flooding. Nat. Geosci. 11:251-257.

Qiu Q, Peng J, Yu X, Chen F, Wang J, Dong F, 2015. Occurrence of microplastics in the coastal marine environment: First observation on sediment of China. Mar. Pollut. Bull. 98:274-280.

Ravit B, Cooper K, Moreno G, Buckley B, Yang I, Deshpande A, Meola S, Jones D, Hsieh A, 2017. Microplastics in urban New Jersey freshwaters: distribution, chemical identification, and biological effects. AIMS Environ. Sci. 4:809-826.

de Souza Machado AA, Kloas W, Zarfl C, Stefan H, Rillig MC, 2017. Microplastics as an emerging threat to terrestrial ecosystems. Glob. Change Biol. 24:1354-1013.

Thompson RC, Moore CJ, vom Saal FS, Swan SH, 2009. Plastics, the environment and human health: current consensus and future trends. Philos. T. R. Soc. B. 364:2153-2166

Tran NH, Reinhard M, Gin KY-H, 2018. Occurrence and fate of emerging contaminants in municipal wastewater treatment plants from different geographical regions-a review. Water Res. 133:182-207. 


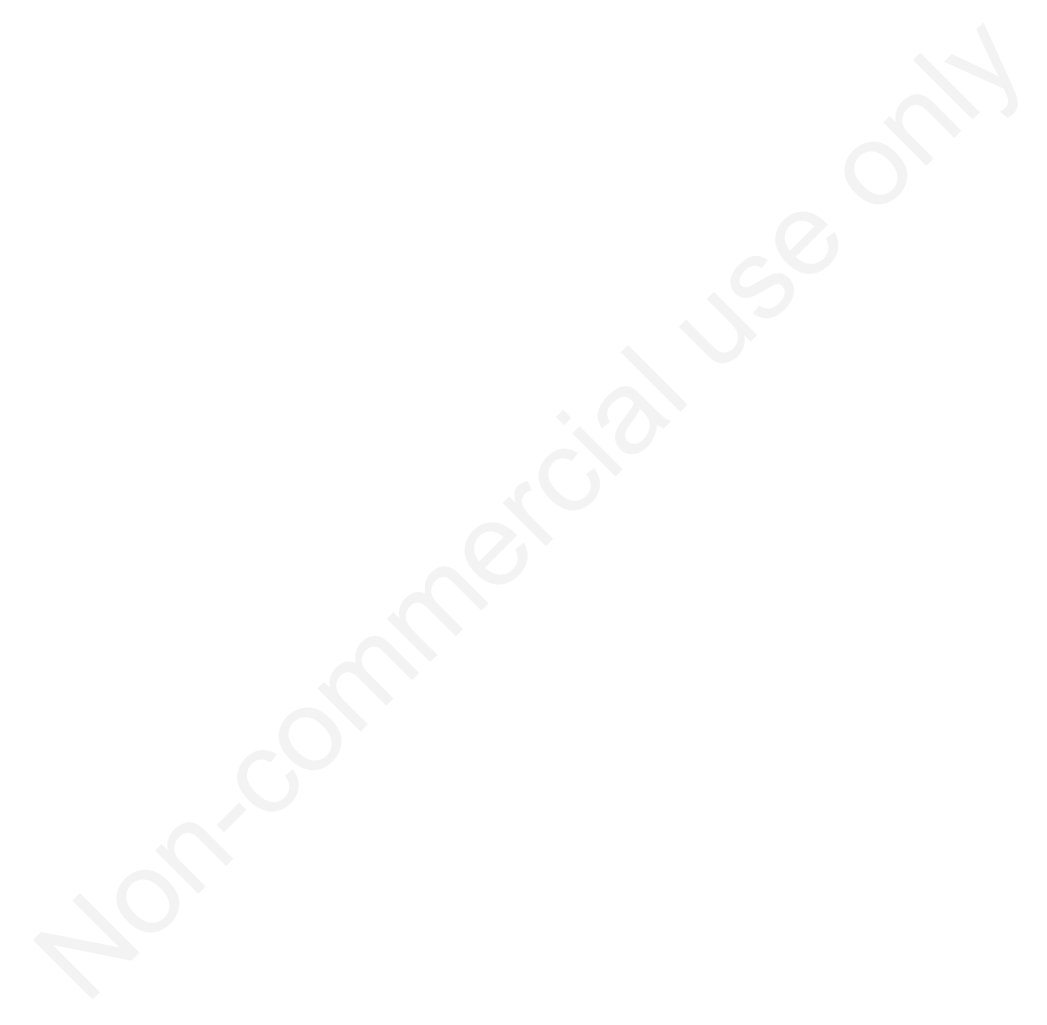

